\title{
Hypertensive Profile of Metastatic Colorectal Carcinoma Patients Treated with Bevacizumab-A Prospective Study
}

\author{
Aravindh S. Anand*, Mintu Mathew Abraham, Jayalekshmi Velayudhan \\ Department of Radiotherapy \& Oncology, Government Medical College, Thiruvananthapuram, India \\ Email: *anandrt2006@yahoo.com
}

Received 17 January 2016; accepted 23 February 2016; published 26 February 2016

Copyright (C) 2016 by authors and Scientific Research Publishing Inc.

This work is licensed under the Creative Commons Attribution International License (CC BY). http://creativecommons.org/licenses/by/4.0/

(c) (i) Open Access

\begin{abstract}
Background: Bevacizumab is a recombinant humanized monoclonal antibody which targets Vasculoendothelial growth factor (VEGF)-A and prevents its binding to VEGF receptor 2 (VEGFR 2). It is approved in the first and second line treatment of metastatic colorectal cancer along with combination chemotherapy. Hypertension is an on-target and a common side effect of the treatment with bevacizumab. The true incidence of bevacizumab induced hypertension and its grading remains unclear due to paucity of prospective studies in this regard. In our institution, majority of metastatic colorectal cancer patients receive bevacizumab along with cytotoxic chemotherapy. In Indian, a context prospective study on this topic is negligible; so we find it to be of high importance. objectives: We have conducted a prospective observational study of all metastatic colorectal patients receiving bevacizumab with chemotherapy and satisfying the eligibility criteria. The objective of the study was to assess the incidence and the pattern of hypertension. Materials \& Methods: Patients receiving bevacizumab with FOLFOX (5 Fluorouracil, Calcium Leucovorin \& Oxaliplatin) or CapeOX (Capecitabine \& Oxaliplatin) chemotherapy for metastatic colorectal cancer were enrolled in the study; blood pressure was monitored before bevacizumab administration and also for the next seven days. This was done for all the chemotherapy cycles. Results: $100 \%$ of patients developed hypertension of any grade and $20 \%$ received treatment as a part of newly detected hypertension. Majority of the patients had grade 1 hypertension. Other grade of hypertension was grade 2 . The blood pressure peaked on $6^{\text {th }}$ day of bevacizumab administration. Conclusions: Hypertension of any grade may develop in bevacizumab treated patients. The incidence is very high and it may reach even up to $100 \%$ if prospectively and meticulously evaluated for longer period of time.
\end{abstract}

\section{Keywords}

Bevacizumab, VEGF, Hypertension, Colorectal Cancer

\footnotetext{
${ }^{*}$ Corresponding author.
}

How to cite this paper: Anand, A.S., Abraham, M.M. and Velayudhan, J. (2016) Hypertensive Profile of Metastatic Colorectal Carcinoma Patients Treated with Bevacizumab-A Prospective Study. Journal of Cancer Therapy, 7, 114-120. 


\section{Introduction}

The field of oncology has improved dramatically in the past few years with the advances in molecular biology of different cancers and the introduction of targeted therapies. It has been three decades since Folk man described his observations on the dependence of tumours on the sustained angiogenesis in tumour survival, growth and metastases. There has been a great effort in drug development and therapeutics targeting angiogenesis.

VEGF is a homodimeric heparin binding glycoprotein which has seminal role in angiogenic process. The VEGF family includes six glycoproteins referred as VEGF-A, VEGF-B, VEGF-C, VEGF-D, VEGF-E, placenta growth factors, PIGF-1 (placenta growth factor) and PIGF-2 [1]. VEGF, unlike other angiogenic factors, is selectively mitogenic for endothelial cells and increase vessel permeability.

VEGF exerts its effects by specific binding to the cell membrane receptors, VEGFR-1, VEGFR-2 or VEGFR3. These receptors are tyrosine kinases, having extracellular immunoglobulin like domains, a transmembrane domain and an intracellular tyrosine kinase domain [2] [3]. In addition, neuropilin 1 (NRP-1) and NRP-2 are co-receptors for specific isoforms of VEGF family members and increase binding affinity of these ligands to their respective receptors [4]. Following the binding of ligand, the receptor undergoes transautophosphorylation with subsequent activation of a cell type dependent signaling cascade processes promoting endothelial cell growth, movement, and survival.

Angiogenesis, sprouting of new blood vessels from the existing ones is critically involved in the pathogenesis and metastases of colorectal cancer. Antiangionesis therapy is an established treatment modality for majority of cancers including colorectal cancer. Bevacizumab is a recombinant humanized monoclonal antibody which targets VEGF-A and prevents its binding to VEGFR2. It is approved in the first and second line treatment of metastatic colorectal cancer along with combination chemotherapy. Bevacizumab in combination with chemotherapy improves progression free survival (PFS) and overall survival (OS) in first and second line setting (Hurwitz et al., 2004 [5]; Kabbinavar et al., 2005 [6]; Saltz et al. 2008 [7]).

Hypertension is an on-target and a common side effect of the treatment with bevacizumab. In the pivotal study by Hurwitz et al. [5], all grades of hypertension was observed in $22.4 \%$ of patients in the bevacizumab containing arm compared with $8.3 \%$ who received chemotherapy alone. Hypertension as a side effect was consistently demonstrated in phase 11/111 clinical trials of bevacizumab. A meta analysis by Loupakis et al. has shown that the relative risk of colorectal cancer patients treated with bevacizumab developing grade $3 / 4$ hypertension is 4.87, $\mathrm{p}=0.0001$ [8].

A number of studies have shown that bevacizumab induced arterial hypertension may correlate with the clinical outcome in colorectal cancer patients [9]. Identification of hypertension as a potential biomarker has increased our understanding of the mechanism of action of anti-angiogenic agents, yet how to implement this knowledge remains uncertain [10].

Several mechanisms are proposed for bevacizumab induced hypertension. It includes rarefaction, decreased number of small arteries and arterioles, increased vascular resistance due to decreased nitric oxide and prostacyclin production etc. [11] [12].

The literature search for studies with regard to hypertension in patients on bevacizumab in Indian population reveals that it is sparse and a prospective study in this topic is negligible. The true incidence of bevacizumab induced hypertension and its grading remains unclear due to paucity of prospective studies in this regard. In our institution, majority of metastatic colorectal cancer patients receive bevacizumab along with cytotoxic chemotherapy. In Indian, a context prospective study on this topic is negligible; so we find it to be of high importance.

Since the study is prospective, we are able to monitor the blood pressure systematically and closely which is highly essential. Hence, we expect our study to be more reliable with regard to the incidence and grading of hypertension. Further continuation studies in this set of patients aim to study hypertension and severity as a biomarker for bevacizumab efficacy in this group of patients.

\section{Objectives and Methods}

This study was approved by the Institutional Human Ethics Committee and informed consent was obtained from the participants in the study. This was a prospective observational study. Those patients receiving bevacizumab and combination chemotherapy from the institution as per the department protocol \& procedures were included in this study. Being an observational study no active intervention was done solely for the purpose of the study. 
The patients were only observed and data collected. The objective of the study was to assess the incidence of hypertension and the pattern of hypertension.

\subsection{Inclusion Criteria}

1) Patients diagnosed as a case of metastatic colorectal cancer radiologically and histopathologically.

2) Patients on bevacizumab with combination chemotherapy FOLFOX or CapeOX.

3) Patients willing for the study and willing for BP monitoring in local hospital after discharge from our institution.

\subsection{Exclusion Criteria}

1) Patients already having uncontrolled hypertension or not on regular antihypertensive.

2) Patients who had not completed at least 6 weeks after surgery.

3) Active bleeding.

4) History of arterial thrombo embolic episodes less than a year.

5) Patients with brain metastases.

6) Patients unwilling for the study or BP monitoring after discharge from our institution.

In our institution, any patient who is diagnosed to have colorectal malignancy first undergoes a staging evaluation as per the standard guidelines. This includes a detailed history \& physical examination, full length colonoscopy \& biopsy, complete blood count, renal and liver function tests, CEA, CT abdomen\& pelvis and CT thorax. After staging work up, those patients who are metastatic CRC will be discussed in multidisciplinary board. Patients who are primarily inoperable are taken up for Bevacizumab and combination chemotherapy. The first line combination chemotherapy in our institution is either FOLFOX or CapeOX. The Bevacizumab dosage is $5 \mathrm{mg} / \mathrm{kg}$ every 2 weeks. Chemotherapy is the standard recommended dosage.

In this study we have prospectively observed these patients if they satisfy the eligibility criteria. After getting enrolled in the study, blood pressure is monitored before bevacizumab administration and also for the next seven days. This was done for all the chemotherapy cycles. The first baseline BP monitoring will be recorded in our institution and the next seven days in the local clinic of the patient. BP was monitored by the same physician all the seven days in the same time, in the supine position and patient relaxed comfortably. BP was monitored using mercury manometer. It was recorded in the BP monitoring chart issued from our hospital. Patients who were already on anti-hypertensives were advised to continue the same. Those patients who were newly diagnosed during the treatment period were advised antihypertensive as per the standard protocol.

Patients were graded based on the JNC 7 (Joint national committee on prevention, detection, evaluation and treatment of high blood pressure) grading criteria of hypertension.

Statistical analysis: patient and demographic details, all laboratory investigations pertaining were entered in the excel sheet 2007. All statistical analyses were carried out using SPSS 18.0 software.

\section{Results}

A total of 30 patients satisfying the inclusion and exclusion criteria were enrolled in the study (Table 1).

The median age of patients was 52 yrs. $23 \%$ of patients were hypertensive prior to the study and they were on antihypertensives and majority of them were on calcium channel blocker. $70 \%$ of the patients received FOLFOX chemotherapy while 30\% received CapeOX. The median number of cycles of bevacizumab was 9 cycles.

$100 \%$ of patients developed hypertension of any grade and $20 \%$ received treatment as a part of newly detected hypertension. Majority of the patients had grade 1 hypertension. Other grade of hypertension was grade 2 . None of the patients stopped bevacizumab usage due to uncontrolled hypertension. The changes in systolic and diastolic hypertension in the first week of bevacizumab are shown in Figure 1 and Figure 2 respectively. Changes in both systolic and diastolic BP were more pronounced in already known hypertensives compared to normotensives prior to bevacizumab (Figure 3 and Figure 4).

The blood pressure peaked on $6^{\text {th }}$ day of bevacizumab administration (Figure 3). The incidence of hypertension was more after two cycles of Bevacizumab (Figure 5 and Figure 6). None of patients showed blood pressure drop after $1 \mathrm{hr}$ of bevacizumab administration while 88\% showed increased blood pressure $1 \mathrm{hr}$ after bevacizumab administration. 


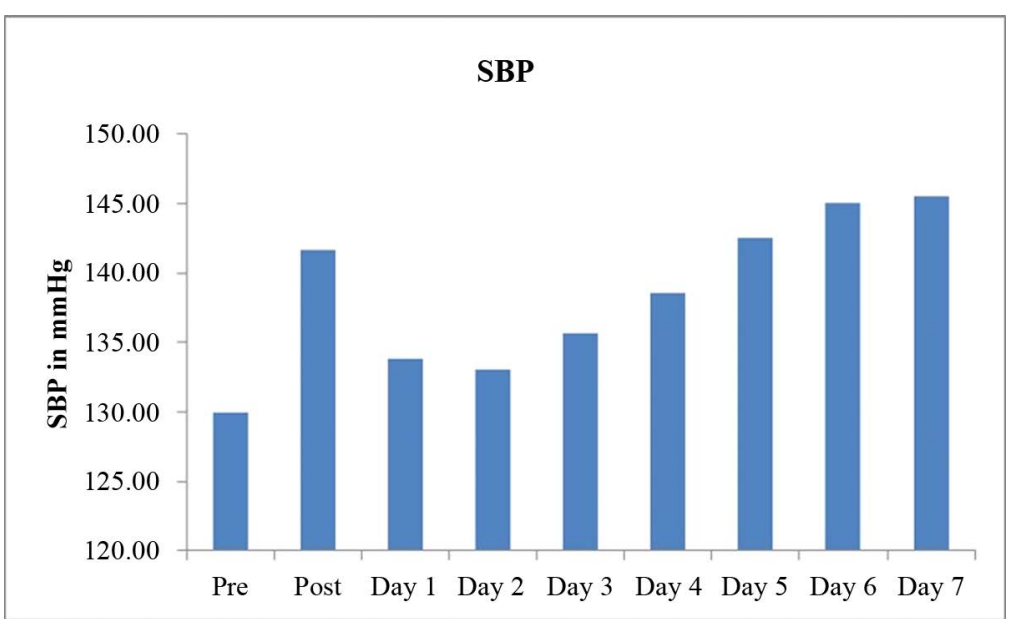

Figure 1. Changes in systolic BP before \& one week after bevacizumab.

Table 1. The baseline patient characteristics.

\begin{tabular}{|c|c|}
\hline Variable & Frequency \\
\hline Median (age yrs) & 52 \\
\hline $\begin{array}{c}\text { Sex } \\
\text { Male } \\
\text { Female }\end{array}$ & $\begin{array}{l}17 \\
13\end{array}$ \\
\hline $\begin{array}{l}\text { Hypertensive before study } \\
\text { Normotensive before study }\end{array}$ & $\begin{array}{c}7 \\
23\end{array}$ \\
\hline $\begin{array}{c}\text { No of metastatic sites } \\
1 \\
>1\end{array}$ & $\begin{array}{c}21 \\
9\end{array}$ \\
\hline $\begin{array}{c}\text { Performance status } \\
0 \\
1 \\
2 \\
3\end{array}$ & $\begin{array}{c}14 \\
12 \\
3 \\
1\end{array}$ \\
\hline $\begin{array}{l}\text { FOLFOX chemotherapy } \\
\text { CapeOX chemotherapy }\end{array}$ & $\begin{array}{c}21 \\
9\end{array}$ \\
\hline Median no of cycles of Bevacizumab & 9 \\
\hline $\begin{array}{l}\text { Antihypertensive treatment before start of bevacizum } \\
\text { Calcium channel blockers } \\
\text { Angiotensin Converting enzyme inhibitor } \\
\text { Beta blockers } \\
\text { Combination }\end{array}$ & $\begin{array}{l}3 \\
1 \\
1 \\
2\end{array}$ \\
\hline $\begin{array}{c}\text { Family history of hypertension } \\
\text { Yes } \\
\text { No }\end{array}$ & $\begin{array}{c}5 \\
25\end{array}$ \\
\hline $\begin{array}{l}\text { Primary tumour } \\
\text { Colon } \\
\text { Recto sigmoid } \\
\text { Rectum }\end{array}$ & $\begin{array}{c}20 \\
2 \\
8\end{array}$ \\
\hline $\begin{array}{l}\text { Metastatic site } \\
\text { Liver alone } \\
\text { Lung alone } \\
\text { Bone alone } \\
\text { Multiple }\end{array}$ & $\begin{array}{l}17 \\
2 \\
2 \\
9\end{array}$ \\
\hline $\begin{array}{c}\text { CEA (ng/ml) } \\
\quad<10 \\
>10\end{array}$ & $\begin{array}{c}3 \\
27\end{array}$ \\
\hline
\end{tabular}




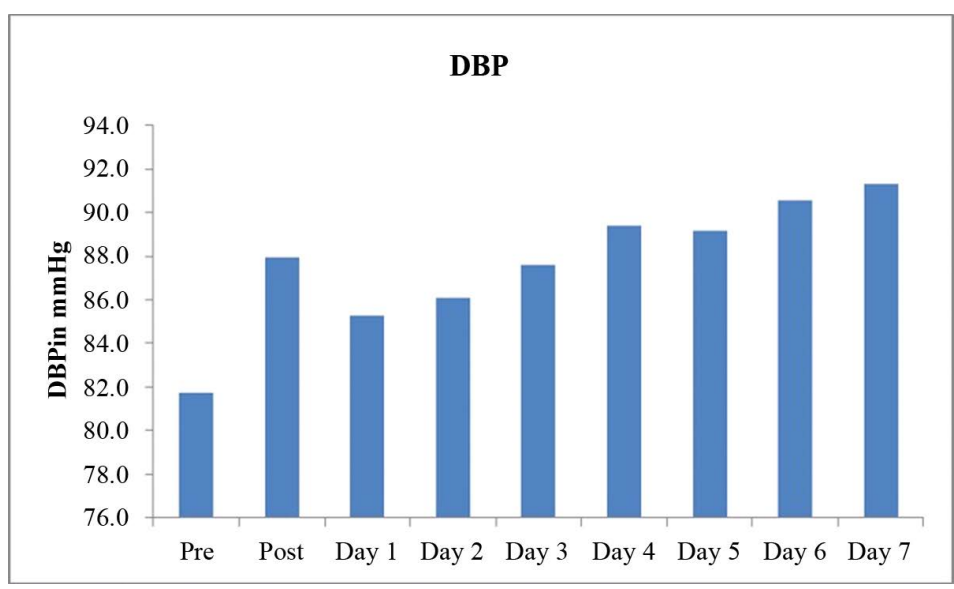

Figure 2. Changes in diastolic BP before \& one week after bevacizumab.

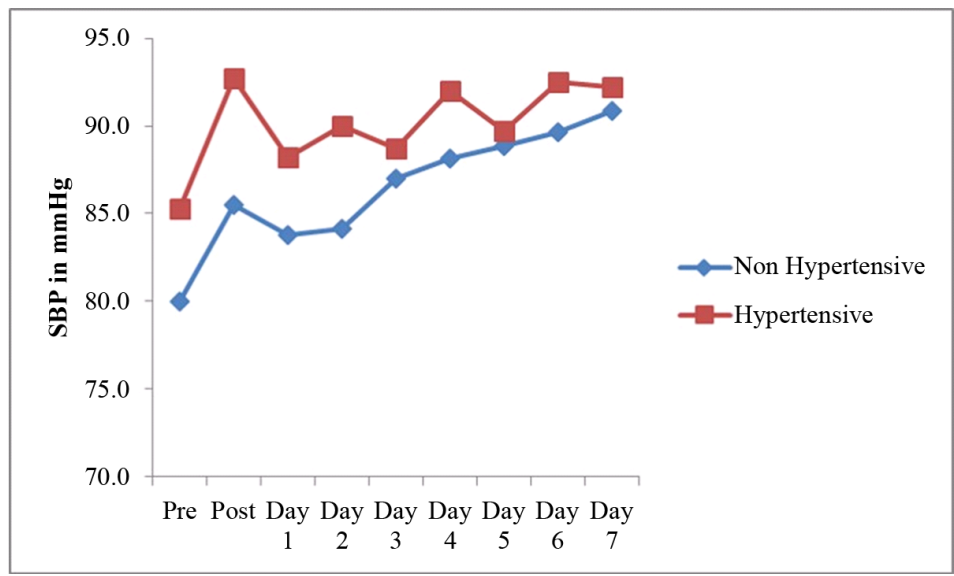

Figure 3. Changes in systolic BP in normotensives and hypertensives.

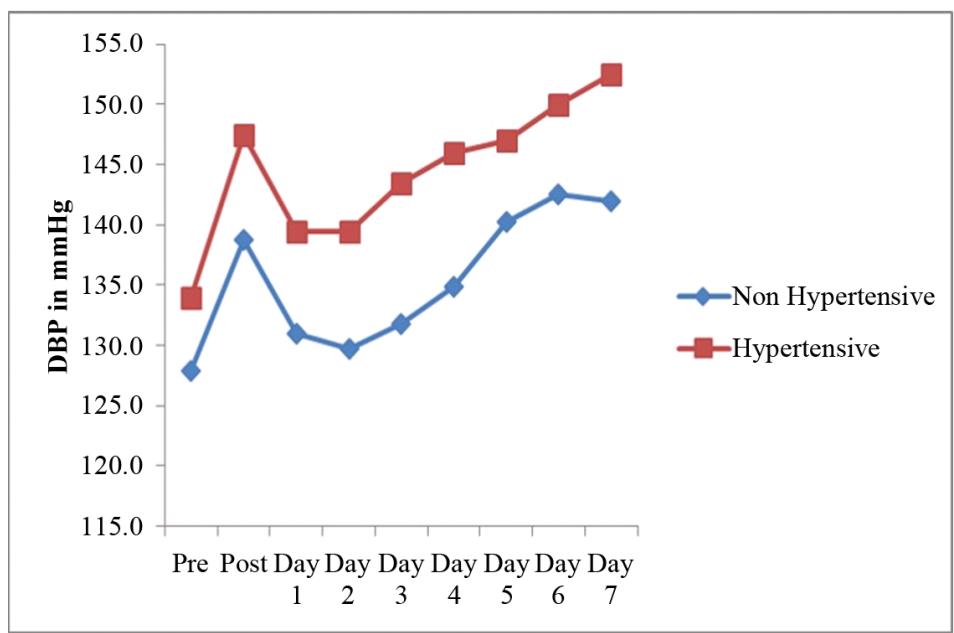

Figure 4. Changes in diastolic BP in normotensives and hypertensives.

\section{Discussion}

Hypertension is a common on target side effect of Bevacizumab. In this prospective study $100 \%$ of the patient developed any grade of hypertension during the treatment period. But only $20 \%$ of patients continued to be 


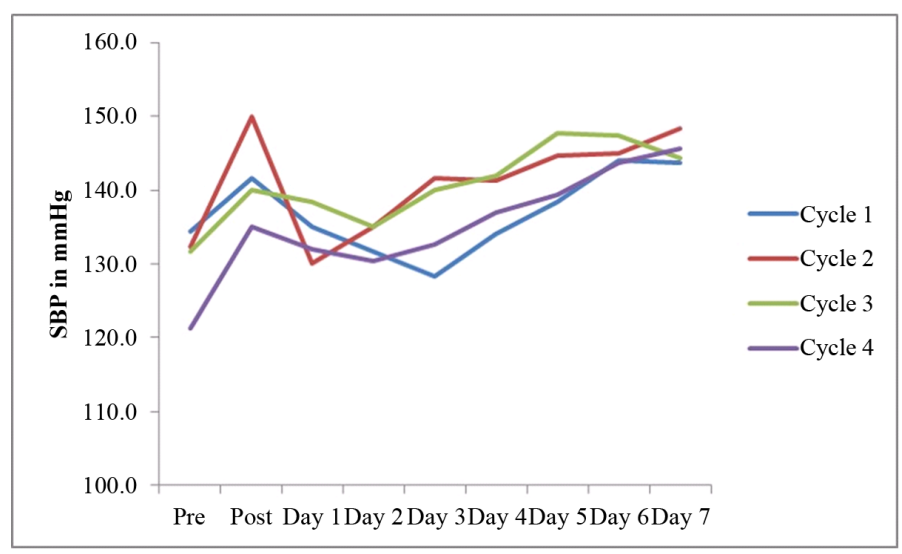

Figure 5. Changes in systolic BP in different cycles of chemotherapy.

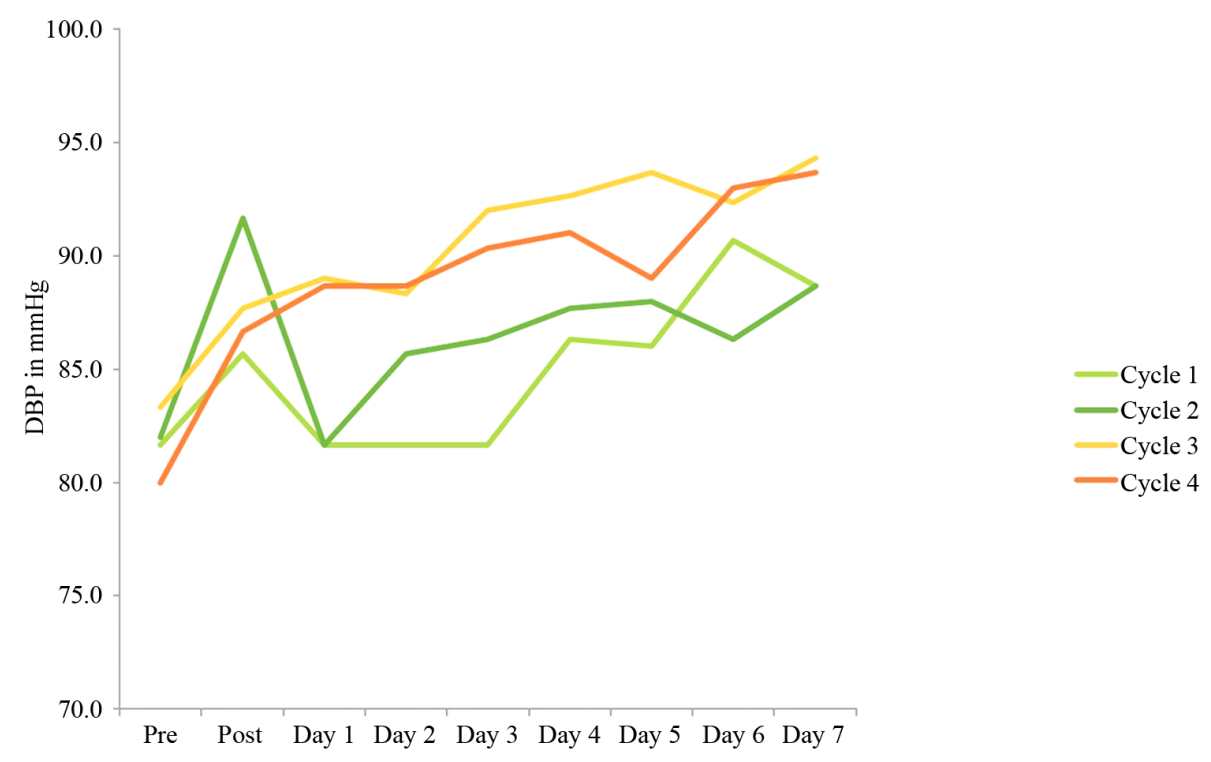

Figure 6. Changes in diastolic BP in different cycles of chemotherapy.

hypertensive and required medications while the remaining $80 \%$ had transient hypertension only. The incidence of hypertension in various trials varies between $19 \%$ to $67 \%$. Our study reveals more incidence of hypertension. This may be due to the fact that majority of other studies were retrospective and not aimed at serial BP monitoring prospectively. In our study the most common grade of hypertension was grade1 while in other studies also it was grade 1. It was observed that hypertension peaks maximum on $6^{\text {th }}$ day of bevacizumab administration. As $100 \%$ of patients developed any grade of hypertension, risk factors like prior history or family history cannot be assessed. In this study, those patients who developed hypertension were treated as per the standard general guideline with angiotensin converting enzyme inhibitor or calcium channel blockers. Blood pressure was monitored closely on discontinuation of bevacizumab and that the doses of antihypertensive drugs were monitored and reduced as appropriate. The objective response rate and hypertension were not assessed in this study as all patients in this study developed hypertension. Assessment of correlation of different grades of hypertension and survival needs longer follow up. Several studies have shown that there is a positive correlation between hypertension and survival. But there are also conflicting results as to whether hypertension is a marker of clinical efficacy to bevacizumab treatment.

\section{Conclusion}

Hypertension of any grade may develop in bevacizumab treated patients. The incidence is very high and it may 
reach even up to $100 \%$ if prospectively and meticulously evaluated for longer period of time. Hence, the important message is that there must be strict monitoring and guidelines of treatment of bevacizumab induced hypertension. It will be better if all patients are provided with BP monitoring chart even after discharge from hospital. This may be helpful in preventing long term effects of hypertension as the incidence is high in this set of patients. Further randomized controlled trials of long term follow up addressing the correlation of treatment response \& survival with different grades of hypertension may be of definite benefit in settling the conflict regarding the same.

\section{Declarations}

Funding: Nil.

Conflict of interest: None Declared.

Ethical approval: The study was approved by the Institutional Human Ethics Committee of Government Medical College, Thiruvananthapuram, Kerala, India.

\section{References}

[1] Ferrara, N., Gerber, H.P. and LeCouter, J. (2003) The Biology of VEGF and Its Receptors. Nature Medicine, 9, 669676. http://dx.doi.org/10.1038/nm0603-669

[2] Yancopoulos, G.D., Davis, S., Gale, N.W., Rudge, J.S., Wiegand, S.J. and Holash, J. (2000) Vascular Specific Growth Factors and Blood Vessel Formation. Nature, 407, 242-248. http://dx.doi.org/10.1038/35025215

[3] Clauss, M. (2000) Molecular Biology of the VEGF and the VEGF Receptor Family. Seminars in Thrombosis and Hemostasis, 26, 561-569. http://dx.doi.org/10.1055/s-2000-13213

[4] Soker, S., Takashima, S., Miao, H.Q., Neufeld, G. and Klagsbrun, M. (1998) Neuropilin-1 Is Expressed by Endothelial and Tumour Cells as an Isoforms Specific Receptor for Vascular Endothelial Growth Factor. Cell, 92, 735-745. http://dx.doi.org/10.1016/S0092-8674(00)81402-6

[5] Hurwitz, H., Fehrenbacher, L., Novotny, W., Cartwright, T., Hainsworth, J., Heim, W., Berlin, J., et al. (2004) Bevacizumab plus Irinotecan, Fluorouracil, and Leucovorin for Metastatic Colorectal Cancer. The New England Journal of Medicine, 350, 2335-342. http://dx.doi.org/10.1056/NEJMoa032691

[6] Kabbinavar, F.F., Schulz, J., McCleod, M., Patel, T., Hamm, J.T., Hecht, J.R., et al. (2005) Addition of Bevacizumab to Bolus Fluorouracil and Leucovorin in First Line Metastatic Colorectal Cancer Results of a Randomized Phase 11 Trial. Journal of Clinical Oncology, 23, 3697-3705. http://dx.doi.org/10.1200/JCO.2005.05.112

[7] Saltz, L.B., Clarke, S., Díaz-Rubio, E., Scheithauer, W., Figer, A., Wong, R., Koski, S., et al. (2008) Bevacizumab in Combination with Oxaliplatin-Based Chemotherapy as First Line Therapy in Metastatic Colorectal Cancer: A Randomized Phase 111 Study. Journal of Clinical Oncology, 26, 2013-2019. http://dx.doi.org/10.1200/JCO.2007.14.9930

[8] Loupakis, F., Bria, E., Vaccaro, V., Cuppone, F., Milella, M., Carlini, P., et al. (2010) Magnitude of Benefit of the Addition of Bevacizumab to First Line Chemotherapy for Metastatic Colorectal Cancer: Meta-Analysis of Randomized Clinical Trials. Journal of Experimental \& Clinical Cancer Research, 29, 58. http://dx.doi.org/10.1186/1756-9966-29-58

[9] Osterlund, P., Soveri, L.M., Isoniemi, H., Poussa, T., Alanko, T. and Bono, P. (2011) Hypertension and Overall Survival in Metastatic Colorectal Cancer Patients Treated with Bevacizumab-Containing Chemotherapy. British Journal of Cancer, 104, 599-604. http://dx.doi.org/10.1038/bjc.2011.2

[10] Dewdney, A., Cunningham, D., Barbachano, Y. and Chau, I. (2012) Correlation of Bevacizumab Induced Hypertension and Outcome in Boxer Study, a Phase 11 Study of Capecitabine, Oxaliplatin (CAPOX) plus Bevacizumab as PeriOperative Treatment in 45 Patients with Poor Risk Colorectal Liver only Metastases Unsuitable for Upfront Resection. British Journal of Cancer, 106, 1718-1721. http://dx.doi.org/10.1038/bjc.2012.152

[11] Chen, I.I.H, Prewitt, R.L and Dowell, R.F. (1981) Micro Vascular Rarefaction in Spontaneously Hypertensive Rat Cremaster Muscle. American Journal of Physiology, 10, 306-310.

[12] Wheeler-Jones, C., Abu-Ghazaleh, R., Cospedal, R., Houliston, A., Martin, J. and Zachary, I. (1997) Vascular Endothelial Growth Factor Stimulates Prostacyclin Production and Activation of Cytosolic Phospholipase $\mathrm{A}_{2}$ in Endothelial Cells via p42/p44 Mitogen-Activated Protein Kinase. FEBS Letters, 420, 28-32. 